\title{
Education as a Process of Social Types' Formation
}

Ildar Nakipovich Valiev

\author{
Kazan Federal University, Russian Federation, 423600, Yelabuga, Kazanskaya Street, 89
}

\section{Doi:10.5901/mjss.2015.v6n4s1p101}

\begin{abstract}
Formation of social types is the aim of the educational system, its role and level of development are defined by the complexity of a social and professional infrastructure, determining the complexity and diversity of social types.
\end{abstract}

Keywords: education, learning, training, development, social type, lifestyle, social portrait, socialization.

\section{Introduction}

Modern analysis of Russian society, especially in a transition period raises the question of new approaches to the study of the laws of its development and education of its representatives (Sedov and Valiev, 2013). Educational environment, understood as a some coherent set of external conditions to the individual providing his social formation in accordance with public expectations, as well as opportunities for his development, contained in a social and spatially-object environment, determines the socialization as the process and the result of human assimilation and appropriation of social life experience. Socialization, in its turn, helps a young person to enter the system of social interaction as its full participant.

They speak more often about the socialization of young people, though the extraordinary social situations, which require for their positive resolution a change (reduction) of social norms, values, behavior patterns, roles, attitudes, customs, cultural traditions, collective ideas and beliefs (for example, as a result of experienced social upheavals or migration), indicate the objective presence some processes of secondary socialization, re-socialization etc. in a social life.

The ambiguity of socialization's meanings as an objective phenomenon, the diversity of its various components, the multiplicity of goals, tasks, social functions, - everything points to its certain "solar" and indeterminable boundaries due to the social complexity and dynamism.

Along with the blurred boundaries of socialization as a social phenomenon and a process, still mechanisms of socialization remain uncertain. At different times different researchers claimed them as follows: heredity (A.Gezell, U.Makdaugall, G.Piaget), environment, along with a childish penchant for imitating someone's behavior (Dzh.Bolduin, Dzh.G.Mid), family and childhood (M. Mid, Jean-Paul Sartre, Z. Freud), socially conditioned learning (Dzh.Uotson, E.Torndayk, K.Holl), social role interaction (Dzh.G.Mid, G.Blumer), internalization of social norms (T. Parsons), etc.

Indicated variety of theoretical positions have a logical explanation. Indeed, as a process the result of the socialization is the acceptance / transfer of social experience from one generation to another, that is, assurance of social activity's continuity in the diversity of its elements and relationships. In this regard, the complexity of socialization's determination fully reflects the complexity of the definition of the society itself, confirmed by many sociologists ( $\mathrm{N}$. Luman, N.Smelzer etc.).

\section{Methods}

Object of study: formation of social types.

Subject of research: education as a process of social types' formation.

Objective: to explore education as a process of formation of social types.

Methods: - theoretical: analysis of the literature, the study and synthesis of teaching experience, methods of abstraction and specification; - empirical: observation, inquiry.

The reliability and validity of the results of the study are provided by a variety of informational sources, comprehensive study of the scientific literature on the survey; validity of the research methodology, using a set of scientific methods, adequate to the object, subject, purpose and objectives of the survey. 


\section{Results}

The position, expressed by A.O.Boronoev and P.I.Smirnov is rather interesting. Challenging the complexity of determination of a society and considering it as a result of neglecting "of some basic requirements that exist in formal logic," they suggest to understand the society as a "people association based on active interaction (i.e., such an interaction, where the exchange of results lies between functionally different groups - V.V.); Society is a system of subjective type, based on human active interaction "(Boronoev and Smirnov, 2003, p.10).

This definition is not entirely new. Karl Marx defined the society through the production relations that can be legitimate, but not exhaustive. All variety of public relations cannot be reduced to productive, functional or activity-related ones. However, in both cases, it is focused not only on the major component of the society, but on the component which performs a system-aggregated function in social relations. That are the production (functional, activity-related) relationships which give a quality integrity to the public, without which it turns into a nominal set of disparate individuals. Reproduction and development of these relations is of a paramount importance in the face of society members of all levels.

Based on the above said and being fully aware that this position greatly simplifies the variety of public relations, let us return to the above definition of a society as a system of interaction between people on the basis of the exchange activity results between different functional groups. Taking into consideration that "social function is a system of socially constructed repeated problems facing the people. By solving those problems people are included into the society" (Shakurov, 1999, p.43), in the future such kind of interactions and relationships will be denoted as functional.

Considered interpretation of society determines socialization not just as the process and the result of people's assimilation and appropriation of social life experience, but as the assimilation of "functionally different" groups' experience (different functional groups - V.V.), and first of all, social and professional ones. This clarification is certainly significantly coarsens interpretation of socialization, but also makes it more operable. In addition, the emphasis on the need to transfer to young people not just a "universal experience", but a whole range of different functional groups' "experience" most closely matches the trend towards constant increasing of social and professional infrastructure's complexity of Russian society.

In the most general sense, social experience is an aggregate system of knowledge and skills needed to implement any social activities in collaboration with other people. Social experience defines: 1) the integration of people into social interaction by assimilation of values, norms and technologies of social activity; 2) differentiation of human due to sociocultural features of social communities and groups; 3) social reproduction of society as a whole so as the various communities (M.I.Bobneva, A.G.Zdravomyslov, E.A.Orlova).

Being individually colored, experience defines some "social type" of a man as an integral characteristic of his stable intellectual and personal peculiarities; on the one hand, they ensure the effectiveness of individual social life activity in its local and general social aspects, and on the other hand, they are provided by a particularly social life.

Externally, the "social type" is similar to the "social portrait" which was intensively developed in sociology since the 70s. XX cen. studying members of various professional groups and social communities. For example, in just the last 1015 years sociology studied social portraits of the consumer of medical services (A.V.Reshetnikov), sports fan (V.A.Viktorov, S.I.Platonov), cohorts of different ages in post-Soviet Russia (LA .Belyaeva), a young entrepreneur (S.V.Grishaev, V.G.Nemirovsky), an employee of the Interior (A.M.Vorobev), etc. However, the concept of "social type" is much deeper.

Of course, the social portrait contains elements of a social type, but is not limited to it because of its dynamism and dependence on external (in relation to the essential characteristics of a man and society) conditions. "Social Portrait" makes an instant slice of a particular group or community's features, characterizing their representatives in their sociotemporal entity "here and now". Changing of a social situation automatically leads to a change in the portrait, in particular, the following parameters, such as satisfaction with the work, labour and living conditions, the level of professional and social activity, education, attitude toward various aspects of social life, income, presence and character of the property, the material and legal status, mood and social orientation, etc. In contrast to the portrait, social type includes the fundamental characteristics and personality features that determine the social existence of a man, but not determined by him.

The category of "social type" also has some intersections with the category of a "lifestyle". According to R.V. Ryvkina, lifestyle is "a combination of several different and interrelated social activities (the most specific to certain categories of people - Valiev I.N.). The chief ones among them are: 1) different kinds of economic, primarily, labor activity - on an enterprise, organization, sphere of individual labour activity, self-employment; in the formal and informal sectors of the economy; in leadership and performing positions; 2) the complex activities in the household - from the taking care of 
children and disabled and meeting daily household needs, in particular, the acquisition of food, clothing and footwear, furniture and vehicles, the purchase and renovation of housing; 3) studies in the field of education (training colleges, institutes, courses, self-study); 4) forms of spending free time (leisure and entertainment, starting with "cultural activities" (going to the theater, watching movies) to the discos, restaurants, going out with friends, etc .; 5) ways to maintain health - sports, participate in hikes, etc .; 6) participation in the political life of the country; 7) labor and territorial mobility - from changing the place of work to the change of a residence; 8) manifestations of deviant behavior, social diseases "(Ryvkina 2001, p.32).

Most often the term "lifestyle" was used to describe the macro-objects - classes (layers) or a certain type of a society. So, the phrase "Soviet way of life" is sufficiently stable, it implies a more or less integrated set of socio-economic, socio-cultural and everyday features of citizens' life in the USSR and the countries of a socialist camp. Similarly, they determined American way of life, Russian population's way of life in conditions of radical reforms (Yu.P.Kovalenko, E.N.Cheremnyh), lifestyle of the Kazakh SSR population (G.O.Asylbekova, M.K.Kulzhanov), urban life image (I.S.Turov), lifestyle of Muscovites (V.Irina, S.V.Tumanov), etc.

A similar approach to the analysis of a lifestyle is peculiar to foreign sociology. So, in D. Treiman's theory the impact on the lifestyle of the existing system of social stratification is analyzed, the characteristics of different people's groups.

In all cases, the lifestyle's studies took into consideration large social groups or societies, averaging their members' characteristics to the maximum. However, analyzing the society from the standpoint of functional interaction of its members, interchange activity results in order to meet the vital needs and interests most effectively, we need to come to a conclusion about the existence of the structure of any large groups' social system, whose representatives have more or less holistic set of individual qualities and features that ensure their effective implementation of a certain kind of social functions. These "coherent sets" having the certainty of quality and in many cases fundamentally different from each other, can be described as "social types".

Social types are non-class and no stratified. Set of individual and personal characteristics peculiar to a representative of a social type, being formed, practically does not change in social functioning. The most important feature of a social type is its invariance. The minister, who made a career starting with an ordinary worker, has not got a greatly changed social type. Moreover the same personal qualities which previously ensured its effectiveness as a worker (or else he would have stayed working), made him the Minister.

This example indicates the fundamental difference between "social type" and "lifestyle." Processes of social mobility may fundamentally change the way of a human life in the transition from one social class to another, when changing professional groups and the like, but his social type is not exposed to such a striking change. A.E. Jakubowski gives the following definition of social types: "It is a certain number of people who are in a similar social situation, having similar social values (i.e. some mental kinship) and social opportunities, implementing a single model lifestyle. ... In theory, there is almost no restrictions in the individual movement from one social type to another one"(Tsvetkova, 2006, p.132).

In the present understanding of the social type, its distinctive features are manifested in determining its invariant qualities of a personality (social and value sets are conservative by definition) and in relation to the way of life. Taking into consideration the nominal similarity of different properties and features of different people we can assume that the social type is characterized not by the presence of its representatives in any unique features, but by some combination of components and various components of a personality. Returning to the definition of a "social type", we note that the possibility of transfer to another social type as immanent (by definition) feature type can be of some doubt, however, it is the subject of an independent research.

The value of the socialization process, the need to manage the reproduction of complex social types that exist in a society and ensure its viability, has led to the emergence of the Institute of Education, acting under the conditions of today as the most important component of social interaction. In view of the above said, the education can be represented schematically as shown below.

Education is inextricably linked with the society, being a deterministic one to social processes on the one hand, and determining them from the other. One can agree with $\mathrm{K}$. Mannheim, stating that:

1. Education forms not human as he is, but a man in this society and for the society.

2. The best educational unit is not the individual but the group. Groups vary in size, objectives and functions. The training different patterns of behavior are produced to be followed by individuals in groups.

3. The objectives of education in a society cannot be adequately understood as long as they are separated from the concrete situations in which each age group falls, and from the social order in which they are formed (Manheim, 1994, p.479). 
From the standpoint of Sociology, Institute of Education is not only intended to ensure the formation of individual representatives of a social change as representatives of different social types, but also the compliance with the overall structure of forming social needs to the types of social and professional infrastructure. Otherwise, we will inevitably come to a mismatch of individual expectations of different groups of the population and the needs of a society in such people who have certain abilities.

Such public attention to education as a form, conditions and results of a human activity often leads to the idealization of education and discordance in the interpretation of the basic pedagogical categories (education, training, parenting), as well as the goals and objectives of education. The last one greatly complicates management of educational activities, primarily at the level of development of national programs and projects.

Analyzing the problems of education in the proper sociological researches, A.M. Osipov and V.V. Tumalev note, in particular, an acute shortage of theoretical and general works, the small number of actual sociological researches of educational problems in the new environment (Osipov and Tumalev, 2004).

\section{Discussion}

Certainly in agreement with the authors that the study of the educational problems should be systematic, and that sociological analysis of the processes of training and education must be accompanied by thorough operationalization and empirical interpretation of the concept of "education" exactly in the context of the subject of sociology, we should be beware of hasty rejection of the educational division into two traditional components - education and training.

Any scientific concept is a perfect reflection of real objects or their relations. Conceptual vacuum or, alternatively, the imposition of concepts is observed in the case where the objective phenomena are not assigned a name or multiple names are assigned simultaneously. Accordingly, the question of whether to use the concepts of "training" and "education" in a sociological practice as a separate entity is defined by the structure of education as an objective social process.

In accordance with Russian legislation, education is a "purposeful process of education and training for the benefit of a man, society and the state, accompanied by a statement of a citizen (students) achievements established by the state educational levels (educational qualifications). By obtaining by a citizen (students) an education means the achievement and confirmation of their specific educational qualification, which certifies the relevant document (Law "On Education in the Russian Federation "). Despite its absolute social value, the definition of education contained in the law, because of its vastness is not operable in terms of disclosure of the educational structure as an objective social phenomenon or process.

The most classical (in terms of modern pedagogy) understanding of the relationship of training and education is presented by Z.G. Nigmatov and A.N. Huziahmetov. They note that education is a "mastery of knowledge, methods of creative activity, the scientific world," i.e., associate training with the formation of the intellectual component of a personality. Education is defined by them (in the narrow and its teaching sense) as "specially organized purposeful formation of social, moral and aesthetic relations" (Nigmatov and Huziahmetov, 1996, p.4).

A similar position is shared by the LI Malenkova, who states that the development of the identity of any person includes two independent sub-processes: "The accumulation of knowledge about the world, skills and their application ... and the second - the process of forming relationships to acquire knowledge and skills for their preparation" (Malenkova, 2002, p.28).

Interpreting the meaning of "relationship" L.I. Malenkova refers to A.V. Petrovsky, and M.G. Yaroshevsky who claim that ratio represents the relative location of various objects and their properties (Malenkova, 2002, p.28). Accordingly, in the context of the educational problems, the formation of human relations is presented as a process of assimilation of the individual (social, moral, aesthetic and others) positions on a wide variety of objects of material and spiritual world - the comparison of himself with the universe.

An approach to the analysis of problems of education from a cultural point of view is also common in pedagogy. For example, I.A. Zimniaya (with a reference to N.S. Rozov) points to the need to consider education as a social institution as a whole whose main function is the function of cultural reproduction (Zimniaya, 2001, p.41). In our opinion, the most vivid embodiment of cultural approach to the education was a pedagogical concept by N.E. Tshurkova. "A culturological view to the educational process - to her mind - solves many teachers' problems. To elect a culturological position in the view of teaching means to analyze the course of events through the prism of existing cultural norms of public life and culture of the highest values ... "( Tshurkova, 2000, p.5).

We should note also a value-based approach to the education which is also reflected in the works of Russian and foreign scientists. The importance of values and value orientations in education B.S.Alishev, A.V. Petrovsky, M.G. Rogov, 
B.C. Shakurov and others greatly emphasize. Thus, according to N.I. Shevandrin, "the purpose of education and development is the formation in the personality such value orientations, attitudes, motives, which would provide assessment and regulation of behavior in accordance with moral and ethical norms accepted in a particular socio-cultural environment. Such education must provide the person an opportunity to make feasible environmental changes in the direction of its development and improvement "(Shevandrin 2001, p.11).

Of course, the differences of outlined above approaches to the education are not critical from a logical point of view. Indeed, the formation of man's relationship to the different manifestations of the universe is impossible without the approval in the personality structure of the corresponding system of values and value orientations that, however, is an integral (and possibly basic) component of culture. It is rather to speak about the allocation of some of the dominant structure in inherently related to each other components of education. At the same time, a brief analysis of the different approaches to the interpretation of the educational essence suggests qualitative determination and independence of the concepts of "education" and "training" reflecting the qualitative certainty defined by these phenomena and processes' concepts.

If training gives people the knowledge how to perform a particular action, education, firstly, answers the question what and why should a person do, and, secondly, creates the necessary personal qualities, such as love to people, responsibility, hard work, etc. That is, what cannot be learned is brought up, and vice versa.

Everything what was mentioned above leads to the conclusion about an inappropriate association of educational and training concepts as having no a fundamental difference. This conclusion is confirmed by the fact that the presence of deep knowledge in any area or for higher (and post collegiate) education is not correlated with the spiritual and moral qualities of a man. Obviously, people may be well educated, but not brought up, and, on the contrary, well trained, but not educated.

Thus, it is advisable to consider education as a certain manmade level corresponding to a certain social type, secured by his participation in the interaction, but are not causally related to learning and education.

The greatest difficulty in understanding the parity / priority of considerable components of the educational process are connected to the objective of education in the education division in the "broad" and "narrow" sense. In a broad sense - it is an objective process that takes place in a society regardless to the wishes and will of a person associated with the formation of a different kind of properties under the action of the elements surrounding social and natural reality (Pityukov 2001, p.22), and biologically oriented to the imitation of adults, copying their actions and behaviors, as well as the processes of adaptive nature.

In the process of social interaction, the personality may be developed without Institute of Education - in any case, a child grows up, learns a certain amount of knowledge (natural learning) and forms the moral and aesthetic attitude (natural upbringing). Evidence of this thesis is repeatedly confirmed by sociological studies, both domestic and foreign scholars. So, E.I.Shevaldina indicates the influence of a parents' lifestyle on the children's lifestyle and health (Shevaldina, 1997), K.Dzhozef's theory of social deprivation states that children are socialized in an appropriate culture of poverty of their parents, etc. Actually, the spontaneous formation was the dominant mechanism of a social type formation and socialization of the vast majority of people in the history of a mankind and is defined in pedagogy as a "family education".

With regard to the process of social formation of a person in addition to the concept of an "education" the concept of "development" and "formation" of the personality are often used, which also require clarification and mutual correlation.

By definition, personal development is a certain type of personal changes, characterized by the direction, irreversibility and regularity ${ }^{1}$. Taking into consideration that the development can be both progressive and regressive, let us point that in the future in relation to the problem of designing the educational environment we will have in mind the progressive development, which corresponds to the tradition of the usage of this concept in the humanities. In this development, we will communicate primarily with the advent of a new structure of the personality qualities and features which were not previously available.

The assignment of any process to the category of developmental processes automatically indicates its determinism caused by several factors and causal relationships. Otherwise, we are faced with changes in the chaotic manner. In this regard, the development of the individual is represented in the form of a hidden, latent process, the beginning of which we see a newborn (and according to some, still in the womb) of a child, and at the end - a person capable of self-responsible life in the conditions of a modern society within the boundaries of a social type.

Integrative approach to the analysis of an individual and society, became widespread in recent decades (A.V.

${ }^{1}$ By virtue of the principle of universal interconnection and interdependence of phenomena and processes, logical (cause-related) process is any process, therefore we will not consider the law as a distinctive feature of the process of personality development. 
Brushlinskii, B.F. Lomov, I.T. Frolov etc.), allowed a fresh look to the interrelation of biological and social development of a personality. In regards to a just remark of M.S. Egorova and T.M. Maryutina, "to replace the traditional confrontation of biological and culturelogical ideas about a man comes more constructive approach, in frames of which the co-evolution of biological and social ... A more adequate thought is that indissoluble unity of the natural and social is characteristic to the personal development "(Egorov and Maryutina, 1992, p.5).

In view of above said it can be pointed that the process of personal development is determined by simultaneous action of a small number of basic biotic and social factors.

The first ones are "responsible" for the general social characteristics of the person, the second are "responsible" for his individual characteristics. For example, the process of socialization is determined by the action of the fundamental laws of social and macro factors that make this process to be essentially similar for all members of a particular society. However, each individual's "history" of socialization includes a list of influences, accidental in its nature, due to the specific and microsocial situations importing their changes to the overall process of a social change. Therefore, if in the most general terms, the processes of social development are common to all people, in detail each person should be on its own, exclusive development path, which makes it absolutely unique.

By biotic factors of development, first of all, we should include genetically inherited human characteristics. Genetic factors direct the development of a person upon the whole, cause the realization of the pledged genotypic program that was "recorded" by generations of his ancestors. These are: the structure of the body, racial peculiarities, susceptibility to certain diseases, personality, temperament, physical strength and endurance, emotional sensitivity, and more. Moreover, " the proposition is put forward that the genotype contains a net basis, firstly, information about the historical past of a man and, secondly, the related program of its individual development adapted to specific social conditions "(Egorov and Maryutina, 1992, p.5).

Thus, we can talk not only about biotic, but also about the social heredity. Nevertheless, social heredity has its own social, rather than biotic nature. Thus, for example, conducted studies have found links between the characteristics of the family (social origin) and educational needs. In particular it was revealed that in most cases, children inherit their parents' social type (Mkrtchyan, 2005). Primarily cultural and educational level of parents in the family and the dominant valueoriented attitudes has a major impact on a social future of their children (ibidem). We can refer to socio inherited qualities the following: orientation for a certain type of behavior, professional interests, the nature and level of development of culture, vocabulary, patterns of interaction, the level of intellectual development and others. All these qualities are due, as a rule, to immediate social environment of a man, and, above all, his family.

Marked social characteristics are not inherited "definitely" - they are absorbed by the baby from the moment of his birth, absorbed unconsciously, without a proper assessment. Accordingly, particularities which develop so occupy an intermediate position between the genetically inherited and socially determined.

In accordance with the principle of conditionality internal changes to external factors, biotic and social heredity is manifested in different ways depending on the external, environmental, environment where people develop. These conditions can also be divided into biotic and social.

Everything that has a different kind of impact on the human body can be referred to biotic environments: the parameters of the air he breathes; quality of the water he drinks; geographical features (latitude, climate, landscape, water, forests, etc.); remoteness from industrial centers and others.

Social conditions include the features of the political system, the local labor market, the availability of a network of cultural and leisure facilities, ethno-cultural and historical features of the environment, the characteristics of the educational infrastructure, livelihood traditions (and in relation to biotic conditions) and several others. The combination of social and biotic disposition of development (expressed in inheritance of features) and the specific conditions under which this development is carried out determines the variability of individual development.

\section{Conclusion}

1. Social formation of a youth is a lengthy process, which is based on education as a form of specially organized, institutionalized activity.

2. The social meaning of education lies in the intergenerational transmission-assimilation of social types i.e. integral invariant sets of qualities and characteristics that determine the effectiveness of social groups' functioning in different areas of a public life; levels of intellectual and social development, reached by some people.

3. Education goes far beyond the institutionalized educational activities. Social formation of a youth as a goal and result of education is an objective process, due to the huge number of factors, some of which are only 
available to be designed within the boundaries of an institutionalized education component.

\section{Acknowledgments}

The work is performed according to the Russian Government Program of Competitive Growth of Kazan Federal University.

\section{References}

Sedov, S., Valiev, I. (2013) Education, Science and Production Integration as a Condition of Dynamics of Social Interaction Stereotypes / Sergey Alekseevich Sedov and Ildar Nakipovich Valiev // World Applied Sciences Journal 27 (Education, Law, Economics, Language and Communication): 325-328, 2013.

Boronoev, A.O. About the concepts of "society" and "social" / A.O. Boronoev, P.I. Smirnov // Sociological studies. - 2003. - \# 8. - P. 3-11.

Shakurov, B.C. Personality: a functional approach / A.D. Shakurov // Kazan Pedagogical Journal "Professional Education". - 1999. - \# 4 (17). - S. 41 - 48.

Ryvkina, R.V. Lifestyle of the Russian population: the social consequences of reforms in the 90s / R.V. Ryvkina // Sociological studies. 2001. - \# 4. - S. 32-39.

Tsvetkova, I.V. How to create a program of educational work: Manual / I.V. Tsvetkova. - M .: Education, 2006. - 176 p.

Manheim K. Diagnosis of our time / Karl Mannheim. - M .: Lawyer, 1994. - 700 p.

Osipov, A.M. Sociology of Education in Russia: Problems and Prospects / A.M. Osipov, V.V. Tumalev // Sociological studies. - 2004. - \# 7. - S. 120-126.

Law "On Education in the Russian Federation" : adopted by the State Duma, 21.12.2012. approved by Federation Council, 26.12.2012.

Nigmatov, Z.G. Theory and Methods of Upbringing / Z.G. Nigmatov, A.N. Huziahmetov. - Kazan, 1996. - 146 p.

Malenkov, L.I. Theory and Methods of Upbringing / L.I. Malenkov. - M.: Russian Pedagogical Society, 2002. - 480 p.

Zimniaya, I.A. Educational Psychology / I.A. Zimniaya. - M .: Logos, 2001. - 384 p.

Tshurkova, N.E. New training / N.E. Tshurkova. - M .: Russian Pedagogical Society, 2000. - 128 p.

Shevandrin, N.I. Psychological testing, correction and personal development / N.I. Shevandrin. - M .: VLADOS, 2001. - 512 p.

Pityukov, V.Y. Fundamentals of educational technology / V.Y. Pityukov. - M .: Publishing house "Gnome and D", 2001. - 192 p.

Shevaldina, E.I. Socio-economic factors of children's health / E.I. Shevaldina // Sociological studies. - 1997. - \# 8. - S. 92-97.

Egorova, M.S. Development as a subject of a psychogenetics / M.S. Egorova, T.M. Maryutina // Questions of psychology. - 1992. - \# 5. P. 5-15

Mkrtchyan, G.M. Stratification of young people in education, employment and consumption / GM Mkrtchyan // Sociological studies. 2005. - \# 2. - S. 104 - 113. 